\title{
Simulation of gigantic jets propagating from the top of thunderclouds to the ionosphere
}

\author{
Lizhu Tong $^{1}$, Kenichi Nanbu ${ }^{1}$, and Hiroshi Fukunishi ${ }^{2}$ \\ ${ }^{1}$ Institute of Fluid Science, Tohoku University, Sendai 980-8577, Japan \\ ${ }^{2}$ Department of Geophysics, Tohoku University, Sendai 980-8578, Japan \\ (Received November 12, 2004; Revised May 12, 2005; Accepted May 12, 2005)
}

\begin{abstract}
A randomly stepped leader propagation model is developed to study gigantic jets, a new type of lightning, connecting thunderclouds to the ionosphere. The thundercloud is considered as one electrode igniting gigantic jets and the ionosphere is assumed as the other. The propagation of stepped leader is considered as a field controlled random growth process. The electric field is produced due to the thundercloud charges and the selfconsistently propagating leader. A leader propagation probability is proposed to determine whether the leader grows at the next step and what the step direction of the leader is in case of growth. The results show that leader propagation spans $\sim 72 \mathrm{~km}$ from igniting position to the ionosphere. The simulation of leader propagation appears to be in agreement with the structure of observed gigantic jets.
\end{abstract}

Key words: Lightning, gigantic jet, leader modeling, thundercloud, ionosphere.

\section{Introduction}

The breakdown of air in long gaps, e.g., several tens of meters, occurs via a growth of a leader from one electrode to the other with a high electrical conductivity (Raizer, 1991). One of the most important features of lightning discharge consists of the random behavior of its trajectory, which is the behavior of leader discharge (Uman, 2001). Niemeyer et al. (1984) have proposed that discharge patterns have fractal dimension. On the basis of this concept, many investigators have developed the fractal models to study streamer and leader discharges in gaseous dielectrics, cloud-to-ground (CG) lightning, lightning above thunderclouds, and so on (e.g., Niemeyer et al., 1989; Femia et al., 1993; Pasko et al., 2000, 2001; Pasko and George, 2002; Petrov and Petrova, 1993, 1999; Petrov et al., 2003; Tong et al., 2005). Pasko and George (2002) have developed a streamer model to study blue jets and blue starters which are considered as positive streamer coronas expanding from the streamer zones of conventional lightning leaders. Petrov and Petrova $(1993,1999)$ and Petrov et al. (2003) have proposed a leader model to study intra-cloud, cloud-to-ground (CG), and cloud-to-ionosphere (CI) lightning discharges. In the study of CI discharges, they used a simplified spherical thundercloud charge to calculate the electric field generated by a thundercloud.

Gigantic jet is a new type of lightning between the thundercloud and the ionosphere (Su et al., 2003). It is a rare discharge phenomenon because the conditions igniting such a giant discharge are not easy to be satisfied, but it is of interest to theoretically study discharge characteristics of gigantic jets. Based on observational data, Su et al. (2003)

Copy right(c) The Society of Geomagnetism and Earth, Planetary and Space Sciences (SGEPSS); The Seismological Society of Japan; The Volcanological Society of Japan; The Geodetic Society of Japan; The Japanese Society for Planetary Sciences; TERRAPUB. suggested that gigantic jets might be negative cloud-toionosphere (NCI) discharges. In a preceding paper (Tong et al., 2004a, hereafter referred to as R1), the initiation of gigantic jets has been analyzed and regarded as negative streamers. This is different from the previous assumption of blue jets which are considered initiated by positive streamers. Research (Pasko and George, 2002) on blue jets have also shown that the simulation results from the fractal model based on negative streamers are not consistent with observations. In the present work it is assumed that the propagation of gigantic jet is a leader discharge process.

In the present work, the thundercloud is considered as one electrode igniting gigantic jets and the ionosphere is assumed as the other. Lightning formation is described by a random growth of leader discharge channels, which is determined by the electrostatic field produced due to thundercloud charges and leader discharge channels. A leader propagation probability is proposed to determine whether the leader grows at next step and what the step direction of the leader is in case of growth. The results yield a threedimensional overall picture of leader propagation, which appears to be in agreement with the structure of observed gigantic jets.

\section{Leader Mechanism of Gigantic Jets \\ 2.1 Critical field for a leader}

Extensive experimental data have been acquired in laboratory situations to support the electrical nature of the formation and development of a leader discharge (Raizer, 1991). The physical processes underlying leader breakdown consist of igniting electrons, leading an avalanche to streamer corona criterion, and evolving streamer corona to leader channel. The formation and development of streamer corona is a part of the leader process. The electric field for ionization threshold can be estimated by $E_{\mathrm{k}}=E_{0} \cdot\left(N / N_{0}\right)$, 
where $E_{0}=3.14 \times 10^{6} \mathrm{~V} \cdot \mathrm{m}^{-1}, N_{0}=2.688 \times 10^{25} \mathrm{~m}^{-3}$, and $N$ is the neutral atmospheric density (Raizer et al., 1998), taken from US Standard Atmosphere (1976). It should, however, be noted that the field $E_{\mathrm{k}}$ is required to ignite a streamer discharge or an avalanche-streamer transition but not for streamer propagation. Research in R1 has indicated that gigantic jets are initiated by negative streamers. The streamer corona is known that in spite of its internal structural complexity involving multiple highly branched streamer channels, its macroscopic characteristics remain relatively stable under a variety of external conditions. The minimum field $-E_{\mathrm{c}}$ required for propagation of negative streamers in air at atmospheric pressure is $\sim-12.5 \mathrm{kV} / \mathrm{cm}$ (Babaeva and Naidis, 1997), which is regarded as a criterion to sustain the propagation of a streamer in the present work.

It is known that the leader process itself is quite complex, and its initiation mechanism and internal physics are not yet fully understood (e.g., Uman, 2001; Raizer 1991). In the present work, we accept the perspective of Petrov and Petrova (1993, 1999) and Petrov et al. (2003). The critical field $\mathrm{E}^{*}$ governing a leader in air is considered to be equal to the field over the streamer zone in the leader. This can be deduced by such a fact that the development of a streamer discharge precedes the formation of a leader. The condition for a streamer-leader transition can be satisfied if the electric field over the length of the streamer zone in a leader exceeds the critical value (Petrov and Petrova, 1999). Since the electric field decreasing with upper distance from the thundercloud is lower than the exponential decrease of the pressure with height, the condition for a streamer-leader transition can be substantially satisfied to ignite an upper lightning discharge. Thus, the critical field for a leader is estimated by the minimum electric field required for streamer propagation (e.g., Petrov and Petrova, 1999; Petrov et al., 2003), i.e., $E^{*}=E_{\mathrm{c}}$ in this work, which can be simply scaled with height proportionally to the neutral atmospheric density (e.g., Pasko and George, 2002). It is assumed that as soon as the electric field around leader tip reaches the $E^{*}$ field, the leader process is developed. The complex process of streamer-to-leader transition is omitted in the present model. The transition from an avalanche to streamer corona refers to $\mathrm{R} 1$.

\subsection{Electric field in leader channel}

We note that the electric field in leader channel cannot be simply scaled with height proportionally to the neutral atmospheric density due to its high electrical conductivity. In stepped leaders, a leader channel is formed from the strongest streamer in the corona at leader tip by a complicated transition process which involves heating and ionization of gas. It is well known that a streamer corona is formed when the field of the space charge increases to the level of the external field, which will occur when a critical number of electrons, $N_{\mathrm{e}} \sim 10^{8}-10^{9}$ at atmospheric pressure (Raizer, 1991, p. 336; Bazelyan and Raizer, 1998, p. 77), is produced in an avalanche. The critical condition can also be represented by an empirical relation (Raizer, 1991, p. 336)

$$
\alpha x \approx 20,
$$

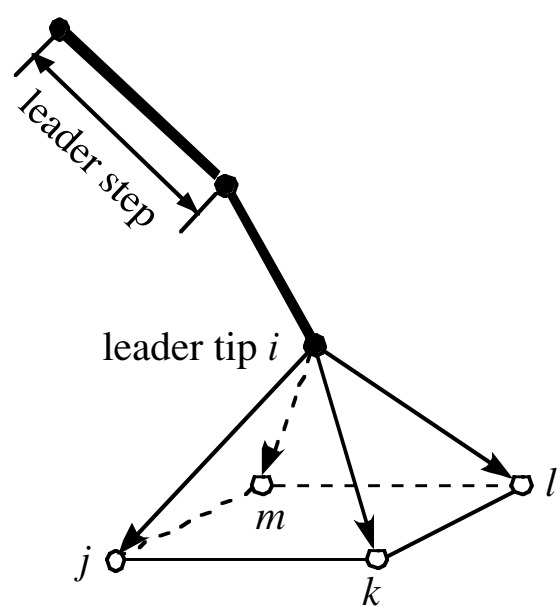

Fig. 1. The schematic of the $E_{i}$.

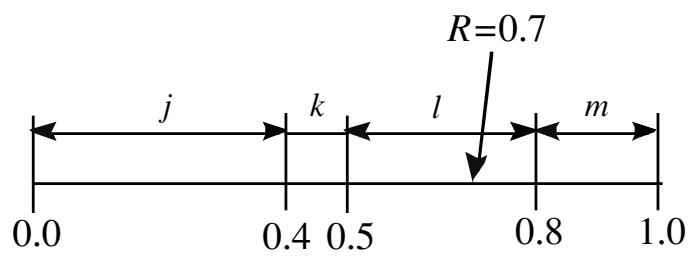

Fig. 2. The method to sample a growth point for leader propagation.

where $x$ is the length of streamer corona and $\alpha$ is the ionization coefficient which can be given by another empirical formula (Raizer, 1991, p. 57)

$$
\begin{array}{r}
\alpha / p=1.17 \times 10^{-4}(E / p-32.2)^{2} \mathrm{~cm}^{-1} \cdot \operatorname{Torr}^{-1} \\
\text { for } E / p \approx 44-176 \mathrm{~V} \cdot \mathrm{cm}^{-1} \cdot \operatorname{Torr}^{-1},
\end{array}
$$

where $p$ is the gas pressure. Once $E / p$ is given, e.g., in the case of the critical situations to form a streamer corona, we have $\alpha \sim p$ from Eq. (2). It is known that the change in density with height is approximately exponential, i.e., $N(z)=N(0) e^{-\gamma z}$, where $\gamma\left(=1.25 \times 10^{-4}\right)$ is the scaling parameter and $z$ is the height above sea level in meters (Petrov and Petrova, 1999). By Eq. (1), the streamer corona $(x)$ under the critical situations becomes longer when $\alpha$ reduces with the decrease of pressure with height, i.e., $x \sim \alpha^{-1}$. The leader length is, however, longer than streamer zone for long leaders. Bazelyan and Raizer (1998) showed some examples of long leaders, e.g., a leader with the length of $11.4 \mathrm{~m}$ consists of streamer zone of 3.6 m. Thus, in the present work we consider the length $L$ of leader channel by $L(z)=L(0) e^{c \cdot \gamma z}$, where $c$ is the parameter that characterizes the variation of leader channel, which is determined by the electric field in leader channel discussed below. It is assumed that the change of the electric field in leader channel with leader length is linear for long leaders, i.e., linearly decreasing as the increase of leader length, such as the experimental data shown in Table 6.1 of Bazelyan and Raizer (1998). Thus, we have $E_{l}(z)=E_{l}(0)[L(0) / L(z)]=E_{l}(0) e^{-c \cdot \gamma z}$. Assigning $\beta=c \gamma$, we obtain $E_{l}(z)=E_{l}(0) e^{-\beta z}$, where $E_{l}(0)=\sim 1 \mathrm{kV} / \mathrm{cm}$ at atmospheric pressure (Raizer, 1991). The parameter $c$ is determined in such a way that $E_{l}(z)$ located in thunder- 
(a)
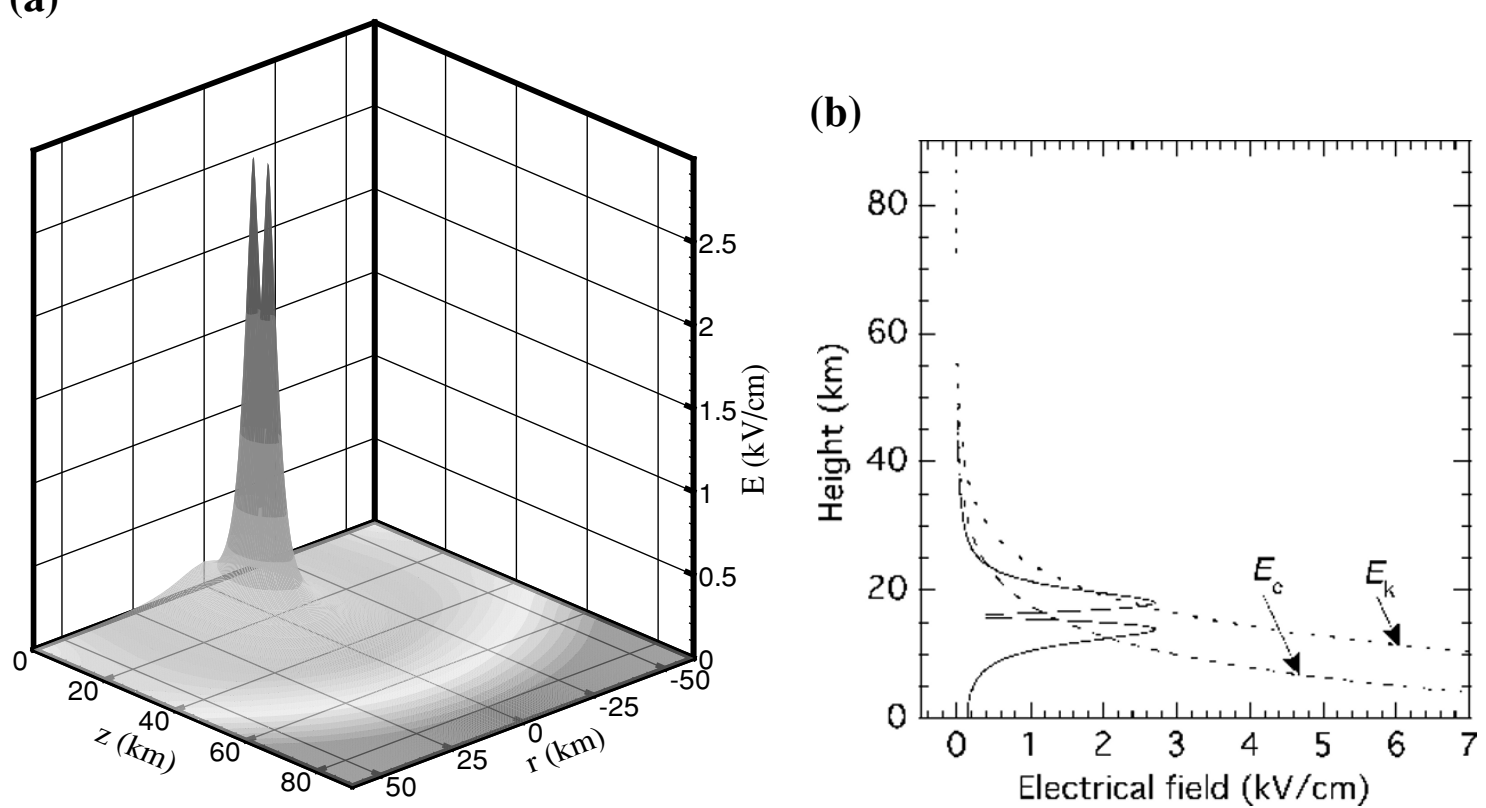

Fig. 3. Electric field distribution at the time that the ionization threshold is reached. (a) Electric field on $r-z$ plane, and (b) Electric field on the axis $(r=0)$.

clouds is consistent with those obtained from the research of intracloud lightning discharges, e.g., Petrov and Petrova (1993). In the present research, $c$ is set 0.8 , which corresponds to the electric field $0.2 \mathrm{kV} / \mathrm{cm}$ in the leader channel in intra-cloud lightning discharges (Petrov and Petrova, 1993). As described above, we propose the method estimating the electric field in leader channel from experimental data obtained in laboratory situations. The estimated field is used to replace the electric field in streamer channel for the original fractal model of Niemeyer et al. (1984). Although the extrapolation exists somewhat risky, to our best knowledge, it is a reasonable compromise of the lack of actual measurements of the electric field in gigantic jets.

\section{Leader Model}

The propagation of the lightning can be considered as a field controlled random growth process, which is a discrete process (Petrov and Petrova, 1999). We schematize the leader propagation as a sequence of connections between the points of a spatial Cartesian lattice. The solution of electric field is divided into two stages: one is for the accumulation of the thundercloud charge and the other is for the propagation of the leader. The former is calculated until the arrival of ionization threshold. The point at which field value reaches ionization threshold is considered as the igniting point of a leader. The potential of this igniting point is then fixed and the discharge is propagated by adding additional links. The continuity equation on the basis of charge conservation law is (Tong et al., 2004b)

$$
\frac{\partial \rho}{\partial t}+\nabla \sigma \cdot \boldsymbol{E}+\rho \sigma / \epsilon_{0}=0
$$

where $\rho$ is the charge density, $\sigma$ is the conductivity, and $t$ is the time. $\boldsymbol{E}$ is electrostatic field governed by

$$
\nabla \cdot \boldsymbol{E}=\left(\rho+\rho_{s}\right) / \epsilon_{0},
$$

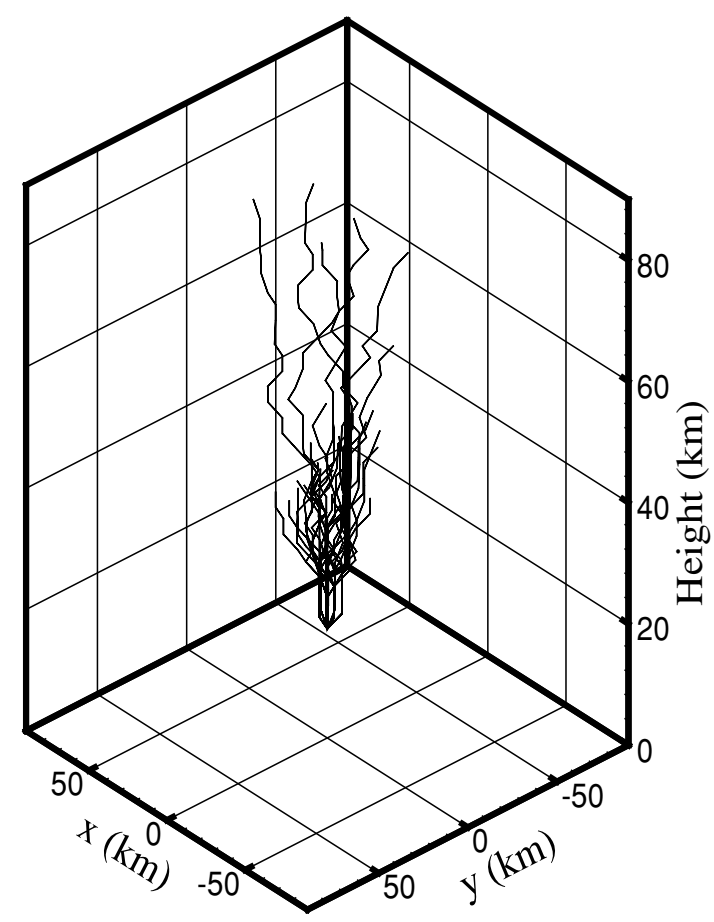

Fig. 4. Leader discharge under the condition that the critical field is reached.

where $\rho_{s}$ is the thundercloud source charge density, i.e. $\rho_{s}$ $=\rho_{-}$, such as that in R1. We consider the electrostatic field at this stage to be axisymmetrical. In the cylindrical coordinate system $(r, z)$, the charge density $\rho_{-}(r, z, t)$ is assumed to be a Gaussian spatial distribution given by $\rho_{-}(r, z, t)=$ $\rho(t) e^{-\left[\left(z-z_{-}\right)^{2}+r^{2}\right] / a^{2}}$, where $z_{-}$is the mean height of negative thundercloud charges and $\rho(t)$ is the charge density corresponding to $Q(t)=Q_{0}[\tanh (t / \tau) / \tanh (1)]$, where $Q_{0}$ is the magnitude of thundercloud charge and $\tau$ is the du- 


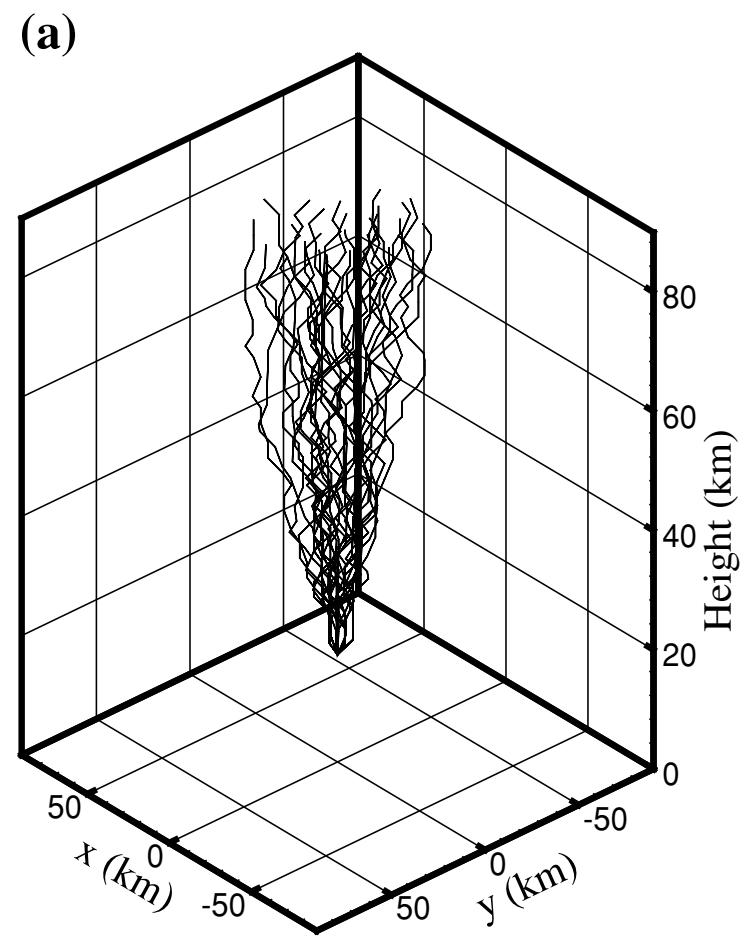

(b)

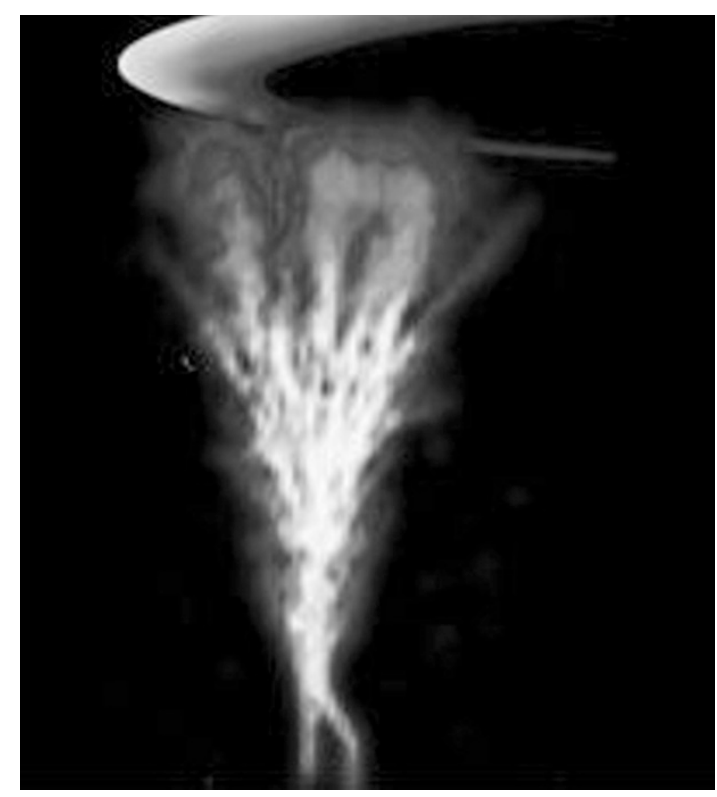

Fig. 5. Leader propagation between thundercloud and the ionosphere. (a) The simulation result based on the present model. (b) Image of a gigantic jet.

ration for the accumulation of thundercloud charge. The detailed discussion related to the charge distribution of thundercloud has been reported in $\mathrm{R} 1$. In this work, we set $z_{-}$ $=16 \mathrm{~km}$ and $a=2 \mathrm{~km}$. Before the ionization threshold is reached, electron conductivity below $60 \mathrm{~km}$ height is low. Therefore the total conductivity $\sigma$ is dominated by ion conductivity, taken by $\sigma=5 \times 10^{-14} e^{z / 6} \mathrm{~km} \mathrm{~S} / \mathrm{m}$ (Dejnakarintra and Park, 1974). The ordinary finite difference method (Potter, 1973) is used to solve Eq. (3). The Fourier transform method and Thomas algorithm (Hockney and Eastwood, 1988) are used to solve Eq. (4).

From the start of the ignition of the leader we solve three-dimensional electrostatic field to satisfy random spatial trajectories of leader propagation. The equation governing three-dimensional electrostatic field is Laplace equation. The field consists of moving boundaries, following leader propagation, which is determined at each growth step of the leader. A three-dimensional isoparametric finite element method (Kagawa, 1994) is used to solve the Laplace equation. At every growth step, a new grid point is linked with the leader channel, which is regarded as an imposed boundary for the recalculation of the electrostatic field. The grid points located in leader channels are assumed to retain the potential which they have acquired until the end of the simulation. The potential drop along leader channels is determined by the electric field $E_{l}$ of leader channel. Next growth point of the leader is randomly chosen from the neighboring points around the leader tip. We parameterize the probability $p_{i}$ for the formation of a leader step as

$$
p_{i}=\left|E_{i}-E^{*}\right|^{\eta} / \sum_{i=1}^{K}\left|E_{i}-E^{*}\right|^{\eta}
$$

where $E^{*}$ is the critical field for a leader, i.e., $E^{*}=E_{\mathrm{c}}$ in this work, $K$ is the total number of the neighboring points around the leader tip, and $E_{i}$ is the average field along the growth direction of the leader.

As seen in Fig. 1, the $E_{i}$ between the points $i$ and $j$ is calculated by $E_{i}=\left(U_{i}-U_{j}\right) / d$, where $U_{i}$ and $U_{j}$ are the potentials at the points $i$ and $j$, and $d$ is the distance between $i$ and $j$. We assume $\eta=1$, following the previous research (Niemeyer et al., 1984; Pasko et al., 2000). Thus, we have $\sum_{i=1}^{K} p_{i}=1$ for $\sum_{i=1}^{K}\left|E_{i}-E^{*}\right|>0$. It is presumed that at each leader step only one point from the neighboring points around the leader tip can become the growth point of the leader. A uniform random number $R$ between 0 and 1 is introduced to choose the growth point (Tong et al., 1998, 2005). As shown in Fig. 1, if there are four possible growth points at the leader tip $i$, i.e., points $j, k$, $l, m$, their relative bond direction probabilities are $0.4,0.1$, $0.3,0.2$, and $R=0.7$, as shown in Fig. 2 , the point $l$ will be considered as next leader growth point. The growth of the leader stops self-consistently when all probabilities become zero.

Since the electric field $E_{l}(=\sim 1 \mathrm{kV} / \mathrm{cm}$ at atmospheric pressure) in leader channel is an order of magnitude lower than the critical field $E^{*}(=\sim 12.5 \mathrm{kV} / \mathrm{m})$. The potential at leader tip sustains a relative higher value by $U_{k+1}=$ $U_{k}-E_{l} \cdot l_{k}$, where $U_{k}$ is the potential at leader tip, $l_{k}$ is the length of leader channel, and $k$ is the leader step. Thus, the propagation of upward electrical discharges from the top of thunderclouds based on leader discharges is much higher than that based on streamer discharges. 


\section{Results and Discussion}

We calculate the quasi-electrostatic field during the accumulation of thundercloud charge on the basis of the solution of Eqs. (3) and (4). Figure 3 shows the distribution of the electric field at the time that the ionization threshold is reached. The thundercloud charge required to reach the ionization threshold is $203.57 \mathrm{C}$. The electric field generated by thundercloud charges just exceeds the critical field $E_{\mathrm{k}}$ for ionization, which is much larger than the critical field $E^{*}\left(=E_{\mathrm{c}}\right)$ for a leader, as shown in Fig. 3(b).

The simulation of leader propagation starts from the time that the ionization threshold is reached. Because the field produced by thundercloud charges is larger than the critical field $E^{*}\left(=E_{\mathrm{c}}\right)$ for a leader, a leader discharge starts to propagate upwards from the position of ionization threshold around the top of thundercloud. The calculation shows that most of the leader discharges terminate below the height of $\sim 40 \mathrm{~km}$, as seen in Fig. 4. Only a few of them propagate over the height and arrive at the ionosphere. Once the leader starts its propagation, the charges will be transported upwards. The accumulation of thundercloud charge can be suppressed not enough to support the leader discharge reaching at the ionosphere. In the present work we use a simple charge distribution of thundercloud in consideration of the effect of the intracloud (IC) discharge or the positive cloud to ground $(+\mathrm{CG})$ discharge. Under the situation the accumulation of thundercloud charge could be maintained. Figure 5(a) gives the calculated result when the thundercloud charge is accumulated up to be $300 \mathrm{C}$. The prevailing leader discharges span the long distance $(=\sim 72 \mathrm{~km})$ from the igniting position around the top of thundercloud to the ionosphere. The leader propagation appears a threedimensional overall picture in agreement with the observed gigantic jets, as shown in Fig. 5(b).

\section{Conclusion}

We develop a randomly stepped leader propagation model to study gigantic jets connecting thunderclouds to the ionosphere. The critical field for a leader and the electric field in leader channel in upper atmosphere are estimated on the basis of experimental data in laboratory situations to compensate for the lack of the measurements. A leader propagation probability is introduced to determine if the leader grows at next step and what the step direction of the leader is in case of growth. The results show that the leader propagation can span $\sim 72 \mathrm{~km}$ from igniting position to the ionosphere. The simulation presents a three-dimensional overall picture of leader propagation, which appears to be in agreement with the structure of observed gigantic jets.

Acknowledgments. The vector computer SX5 at the Institute of Fluid Science of Tohoku University is used in the present simulation. We would like to express our gratitude to Mr. Jean-Claude Vérité of Electricité de France for helpful discussion for this research.

\section{References}

Babaeva, N. Yu. and G. V. Naidis, Dynamics of positive and negative streamers in air in weak uniform electric fields, IEEE Trans. Plasma Sci., 25, 375-379, 1997.

Bazelyan, E. M. and Yu. P. Raizer, Spark Discharge, CRC Press, Florida, 1998.

Dejnakarintra, M. and C. G. Park, Lightning-induced electric fields in the ionosphere, J. Geophys. Res., 79, 1903-1910, 1974.

Femia, N., L. Niemeyer, and V. Tucci, Fractal characteristics of electrical discharges: experiments and simulation, J. Phys. D: Appl. Phys., 26, 619-627, 1993

Hockney, R. W. and J. W. Eastwood, Computer Simulation using Particles, IOP Publishing Ltd, Bristol, 1988.

Kagawa, Y., FEM Program 1, Morikita Shuppan Co., Tokyo, 1994 (in Japanese).

Niemeyer, L., L. Pietronero, and H. J. Wiesmann, Fractal dimension of dielectric breakdown, Phys. Rev. Lett., 52, 1033-1036, 1984.

Niemeyer, L., L. Ullrich, and N. Wiegart, The mechanism of leader breakdown in electronegative gases, IEEE Tran. Electr. Insul., 24, 309-324, 1989.

Pasko, V. P., U. S. Inan, and T. F. Bell, Fractal structure of sprites, Geophys. Res. Lett., 27, 497-500, 2000.

Pasko, V. P., U. S. Inan, and T. F. Bell, Mesosphere-troposphere coupling due to sprites, Geophys. Res. Lett., 28, 3821-3824, 2001.

Pasko, V. P. and J. J. George, Three-dimensional modeling of blue jet and blue starters, J. Geophys. Res., 107, 1458, doi:1029/2002JA009473, 2002.

Petrov, N. I. and G. N. Petrova, Physical mechanisms for intra-cloud lightning discharges, Tech. Phys., 38, 287-290, 1993.

Petrov, N. I. and G. N. Petrova, Physical mechanisms for the development of lightning discharges between a thundercloud and the ionosphere, Tech. Phys., 44, 472-475, 1999.

Petrov, N. I., G. N. Petrova, and F. D'Alessandro, Quantification of the probability of lightning strikes to structures using a fractal approach, IEEE Trans. Diele. Electr. Insul., 10, 641-654, 2003.

Potter, D., Computational Physics, John Wiley, New York, 1973.

Raizer, Yu. P., Gas Discharge Physics, Springer-Verlag, Heidelberg, 1991.

Raizer, Yu. P., G. M. Milikh, M. N. Shneider, and S. V. Novakovski, Long streamers in the upper atmposphere above thundercloud, J. Phys. D: Appl. Phys., 31, 3255-3264, 1998.

Rowland, H. L., Theories and simulations of elves, sprites and blue jets, $J$. Atmos. Terr. Phys., 60, 831-844, 1998.

Su, H. T., R. R. Hsu, A. B. Chen, Y. C. Wang, W. S. Hsiao, W. C. Lai, L. C. Lee, M. Sato, and H. Fukunishi, Gigantic jets between a thundercloud and the ionosphere, Nature, 423, 974-976, 2003.

Tong, L., F. X. Zgainski, J. C. Vérité, P. Thomas, and A. Comte, Discharge simulation in $\mathrm{SF}_{6}$, Proc. of the XIIIth Int. Symposium on Physics of Switching Arc, Brno, Czech Republic, 1, 29-32, 1998.

Tong, L., K. Nanbu, and H. Fukunishi, Numerical analysis of initiation of gigantic jets connecting thunderclouds to the ionosphere, Earth Planets Space, 56, 1059-1065, 2004a.

Tong, L., K. Nanbu, Y. Hiraki, and H. Fukunishi, Particle modeling of the electrical discharge in the upper atmosphere above thundercloud, $J$. Phys. Soc. Jpn., 73, 2438-2443, 2004 b.

Tong, L., K. Nanbu, and H. Fukunishi, Randomly stepped model for upward electrical discharge from the top of thundercloud, J. Phys. Soc. Jpn., 74, 1093-1095, 2005.

Uman, M. A., The Lightning Discharge, Dover, Mineola, N. Y., 2001.

US Standard Atmosphere 1976, NOAA-S/T 76-1562, US Government Printing Office, Washington, DC, 1976.

L. Tong (e-mail: tong@ifs.tohoku.ac.jp), K. Nanbu, and H. Fukunishi 